\title{
Performance Analysis of Photometric Strain Biosensor for Bones using Artificial Neural Network
}

\author{
Preeti Singh \\ UIET, Panjab University \\ Chandigarh
}

\author{
H.M. Rai \\ GIMT, Kurukshetra University \\ Kurukshetra
}

\begin{abstract}
Osteoporosis is a disease of bones. It leads to an increased risk of fracture. The improvement in biosensor for measuring the strain on bones is required. A photometric biosensor is modeled. It is simulated. The performance of the biosensor is analyzed using Artificial Neural Network (ANN) in terms of layers of neural network. Number of epochs/iterations are carried out. The performance is analyzed in terms of mean square error (mse). The percentage accuracy of sensor is obtained as $93 \%$.
\end{abstract}

\section{General Terms}

Photometric, osteoporosis, Artificial Neural Network, accuracy,

\section{Keywords}

biosensor, bones, mean square error, microbend, layer, strain.

\section{INTRODUCTION}

Osteoporosis is a disease of bones. It leads to an increased risk of fracture. It is affecting a large population irrespective of the age. The form of osteoporosis most common in women after menopause is called, "Primary Type 1" or Postmenopausal Osteoporosis. "Primary Type 2" osteoporosis also called Senile Osteoporosis occurs after age 75 . It is seen at a ratio of $2: 1$ in both men and women. Finally, "Secondary Osteoporosis" may arise at any age. It affects males and females equally.

There are number of biosensors in existence to make the diagnosis of such severe health problems. Most of the researches like [1],[2],[3] have focused on using electrochemical and magnetostatic transducers for the measurement of strain. Few of the researchers have emphasized in the field of photometric sensors [4],[5,[6]. The photometric sensors have the advantage of being chemically inert. They do not cause thrombosis. Moreover, these are light flexible and EMI/RFI immune. These biosensors are electrically inert. These reduce the fear of patients in terms of shocks. A work has been reported in the area of optimizing the biosensors for different medical applications using Artificial Neural Network (ANN) [7], [8], [9], [10]. Starodub et al developed surface plasmon resonance optical bioosensors for Express-Diagnostics of Acute Viral Infection and Mycotocsicosis[11]. A very little work has been reported in the area of optimizing the fiber-optic biosensor for strain measurement in ortho applications using ANN. In this paper, an attempt has made to analyze the performance of an optical biosensor using ANN by varying the number of layers.

\section{ARTIFICIAL NEURAL NETWORK}

Basic structure of Artificial Neural Network (ANN) is shown in Fig. 1. It is considered as parallel computational model with varying degrees of complexity. A very important feature of neural networks is their adaptive nature. It is comprised of densely interconnected adaptive processing units. The "learning by example" replaces traditional "programming" in solving problems. This feature makes this computational model very appealing in application domains, where one has little or incomplete understanding of the problem to be solved but where training data is readily available. A neural network consists of three basic types of layers;

(i) an input layer,

(ii) an output layer and

(iii) hidden layers.

These are shown in Fig. 1.

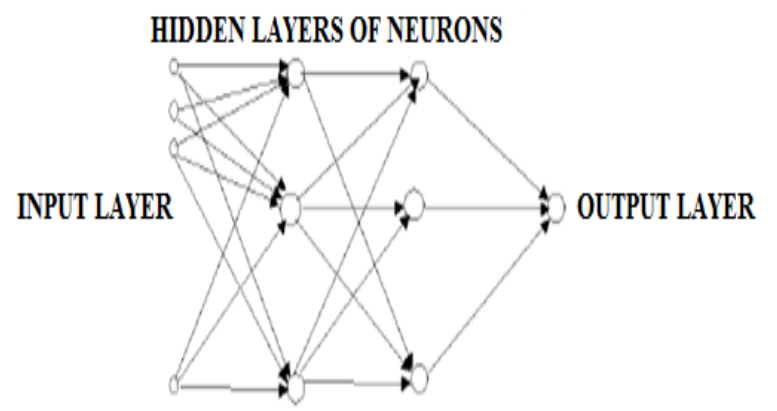

Fig 1: Basic structure of ANN showing various layers

The neural network with one layer of neurons consists of output layer only, no hidden layers. The simplest network consists of just one neuron with the function

$$
g=\sum_{j=0}^{m} w_{j} x_{j}, a
$$

where $\mathrm{g}=$ activation function

$$
\begin{aligned}
& \mathrm{w}=\text { vector of synaptic weights } \\
& \mathrm{x}=\text { vector of inputs } \\
& \mathrm{a}=\text { absolute inhibition term }
\end{aligned}
$$

It is chosen to be an identity function as

$$
\mathrm{g}(\mathrm{v})=\mathrm{v} \text { for all } \mathrm{v},
$$

where, $\mathrm{v}=\sum_{j=0}^{m} \mathrm{w}_{\mathrm{j}} \mathrm{x}_{\mathrm{j}}$

$$
\mathrm{V}=\text { weighted sum of } \mathrm{m} \text { inputs. }
$$


In the present research, the output of the network is linear function of the input vector $\mathrm{x}$ with components $\mathrm{x}_{\mathrm{j}}$. If the dependent variable $\mathrm{y}$ is modeled using multiple linear regressions, it can interpret the neural network as a structure that predicts a value $\widetilde{y}$ for a given input vector $\tilde{x}$ with the weights being the coefficients. If these weights are chosen to minimize the mean square error using observations in a training set, these weights would simply be the least squares estimates of the coefficients. The weights in neural nets are also often designed to minimize mean square error in a training data set. However, there is, a different orientation in the case of neural nets: the weights are"learned". The network is presented with cases from the training data one at a time and the weights are revised after each case in an attempt to minimize the mean square error. This process of incremental adjustment of weights is based on the error made on training cases. It is called, 'training' the neural net. [12]

\section{METHODS AND MATERIALS}

Fiber Optic biosensors have several advantages over the available electrochemical and magnetostatic biosensors. Strain on bones can be measured more accurately with the improvement in biosensors. Such an improvement is being achieved with the design of fiber optic microbend biosensor

\subsection{Block Diagram / Flow Chart}

The block diagram of the proposed fiber optic biosensor is developed and is shown in Fig.2. It consists of various sections like the source, multimode fiber, detector, preamplifier, A/D converter, microcontroller and the LCD display. The source or the optical transmitter converts the electrical signal into the optical signal. Optical signal is fed to the Multi Mode Fiber (MMF). MMF connects the transmitter to the detector or the fiber optic receiver. The MMF is proposed to be bonded with the bone. The strain of the bone is to be measured. The signal from detector is fed to the preamplifier. The output of preamplifier is fed to A/D converter. It is further connected to microcontroller. When the strain on the fiber is applied the output in terms of attenuation can be viewed on the LCD display.

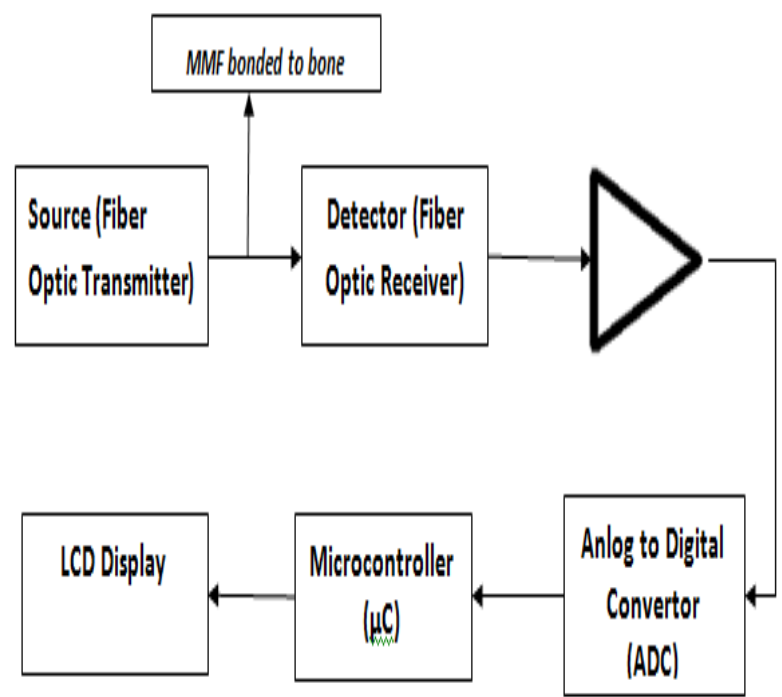

Fig. 2: Block Diagram of the proposed Fiber Optic Biosensor

\subsection{Hardware Model}

On the basis of the block diagram shown in Fig. 2, a model of fiber optic biosensor is designed. Such model is shown in Fig.3. It is fabricated in the project lab of UIET Chandigarh. The accuracy of the fabricated sensor is checked with the aid of strain gauge sensor. It is found that as the with the increase in bend or strain on fiber the loss or attenuation changes proportionately.

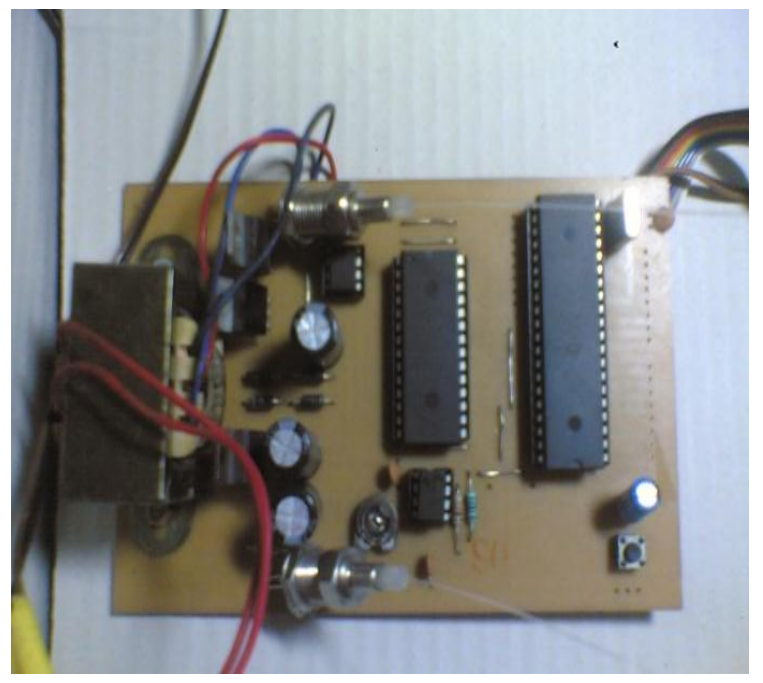

Fig 3: Data acquisition system for biosensor

\subsection{Software Model}

On the basis of the system fabricated in section 3.1, a simulink model is designed. MATLAB has been used for simulation work. The ANN based test bench has been developed for optimization of fiber optic biosensor for strain measurement in ortho applications using MATLAB. Then subsequently iterations have been performed to analyze the performance of the simulink model by considering the concept of layers. The strain versus attenuation is further linearised using the data collected. The linear graph is shown in Fig. 4 [13].

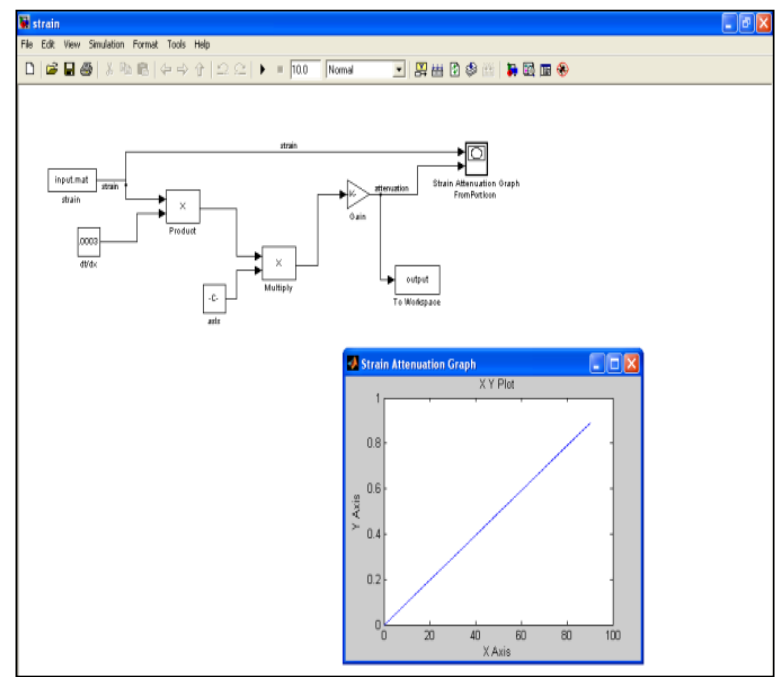

Fig 4: Response of model of biosensor Strain vs. attenuation response 
A series of simulations have been carried out on the ANN based biosensor. These use the concept of layers. On this basis, the performance of the system has been analyzed. First of all, the analysis is being made for the designed simulink model based on ANN with one layer. The neural network training tool is shown in Fig. 5. It depicts the various sections of ANN simulation process i.e. neural network system, algorithms, progress and plots. Neural network system comprises of various blocks showing input output and hidden layer. Algorithm section depicts the model used to train data, performance evaluation parameter i.e. mean square error (mse) and data division section i.e. random in present case. Progress section showing various parameters to analyze the progress of the system i.e. Epoch, time, performance, gradient, $\mathrm{Mu}$ and the validation checks. Plots section consists of performance, training states and regression subsections.

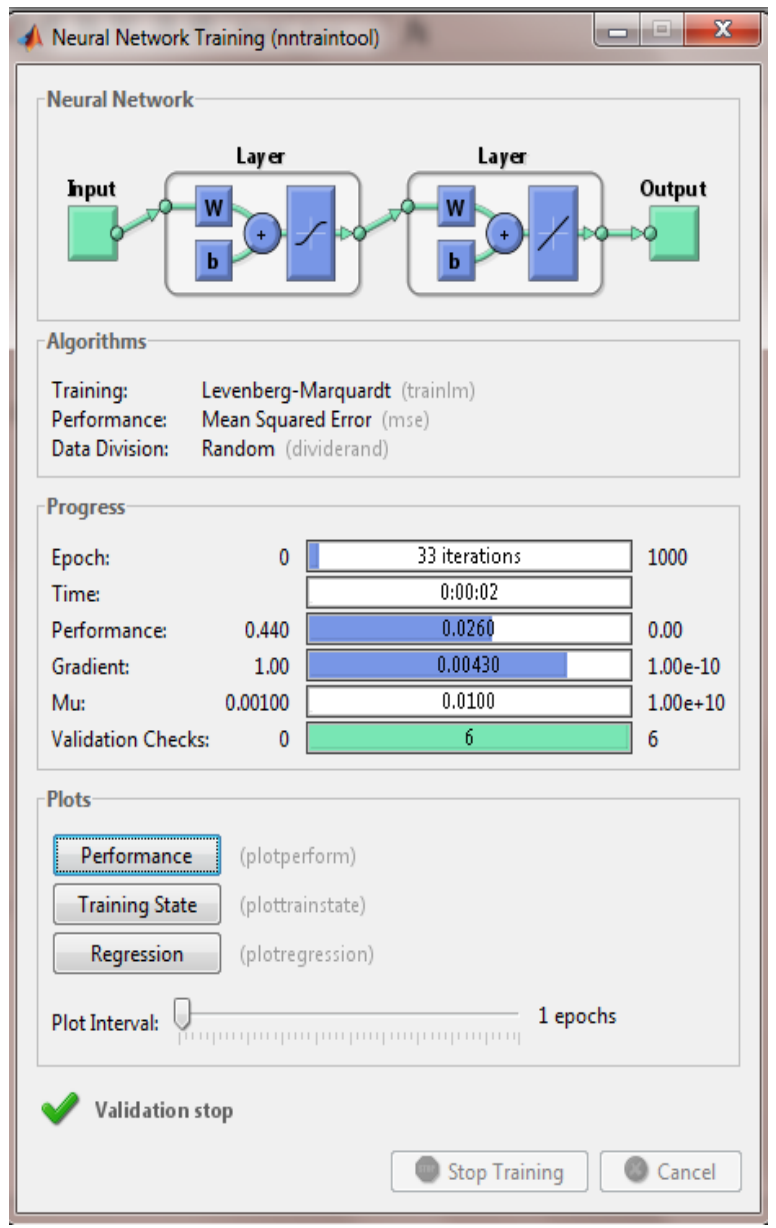

Fig 5: Neural network training tool showing various parameters of progress

\section{RESULTS AND DISCUSSION}

In the present research work, ANN based biosensor is considered in terms of a single layer of neural network. Number of iterations is being carried out. It is found that number of epochs carried out is 33 (Fig. 5) when neural network trained biosensor with one layer is considered.

The performance of training state in terms of gradient, $\mathrm{Mu}$ and validation checks with respect to number of epochs is shown in Fig. 6. The respective values of gradient, $\mathrm{Mu}$ and validation check at epoch 33 are $0.0042956,0.01$ and 6 respectively. The system performance is analysed in terms of number of epochs versus mse. It is shown in Fig. 6. The graph shows trends of trained, validation and test data in terms of epochs versus mse. It is clear that with the increase in number of epochs the mean square error decreases for all trained, validation and test data with slight difference in the slope. The best validation performance in terms of mse is 0.043231 at epoch 27. On the basis of parametetric performance the percentage accuracy of the system designed comes out to be $93 \%$.

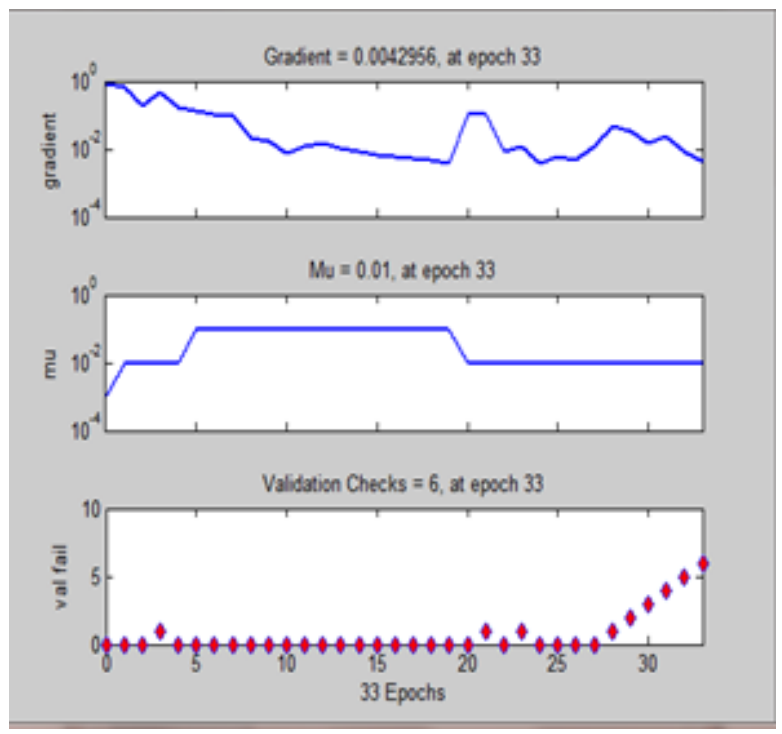

Fig 6: Number of Epochs vs. training state parameters

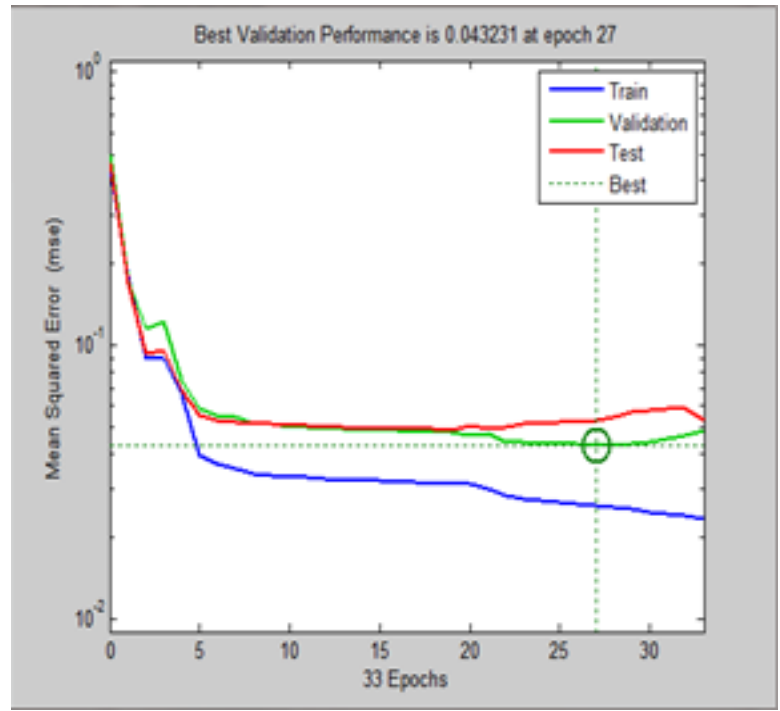

Fig 6: Number of epochs vs. mean square error

\section{CONCLUSION}

With the improvement in biosensor for measuring the strain on bones it will be possible to more accurately detect the onset of osteoporosis. For such an improvement, a model of microbend photometric biosensor is designed and fabricated. The performance of the present biosensor is analyzed using 
ANN in terms of layers of neural network. The trained, validated and test data is analyzed as number of epochs vs. mean square error. The best validation performance in terms of mse is obtained as 0.043231 . The percentage accuracy of the ANN based photometric strain biosensor trained using one layer comes out to be $93 \%$.

The performance of the ANN based biosensor shall be analyzed by varying the number of layers.

\section{REFERENCES}

[1] Wen, Y.-H.; Yang, G.Y.; Bailey, V.J.; Lin, G.; Tang, W.C.; Keyak, J.H.; 2005, "Mechanically robust microfabricated strain gauges for use on bones," Micro technology in Medicine and Biology,. 3rd IEEE/EMBS Special Topic Conference on , pp. 302- 304, 12-15 May, 2005.

[2] Oess, N. P.; Weisse, B.; Nelson, B. J.; 2009, "Magneto elastic Strain Sensor for Optimized Assessment of Bone Fracture Fixation,".Sensors Journal, IEEE , vol.9, no.8, pp.961-968.

[3] Harada, A.; Sakai, R.; Miyasaka, K.; Suzuki, H.; Ohtsuka, T.; Yoshikawa, Y.; Matsuyama, J.; Ohnishi, I.; Nakamura, K.; 2006, "2A-1 A New Method for Measuring Bone Strength using Echo-Tracking," Ultrasonic Symposium,. IEEE , pp.13-16, 2-6 (Oct. 2006)

[4] Abdullah, J.; Ahmad, M.; Lee Yook Heng; Karuppiah, N.; Sidek, H.; 2005, "Fabrication of an optical biosensor based on immobilized MBTH and tyrosinase for determination of phenolic compounds," Sensors and the International Conference on new Techniques in Pharmaceutical and Biomedical Research, Asian Conference on, pp. 103- 106, 5-7 (Sept. 2005)

[5] Swati, M.; Srivastava, R.; 2008, "Alginate Microspheres Comprising Multilayered Assemblies of Cresol Red and Polyelectrolytes Towards an Optical Urea Biosensor," Nanotechnology,. NANO '08. 8th IEEE Conference on , pp.733-736, 18-21 (Aug. 2008)

[6] Premachandran, C.S.; Sheng, K.C.W.; Singh, J.; Teo, J.; Xu Kingship; Chen Nanguang; Sheppard, C.; Olivo, M.; 2007, "Design, Fabrication and Assembly of an Optical Biosensor Probe Package for OCT (Optical Coherence Tomography) Application," Electronic Components and
Technology Conference,. ECTC '07. Proceedings. 57th., pp.1556-1560, May 29 2007-June 12007

[7] Ling Gao; 2011, "Processing of simulated biosensor data using artificial neural networks," IT in Medicine and Education (ITME), International Symposium on , vol.1, pp.27-30, 9-11 (Dec. 2011)

[8] Guo-Zhu Chu; Chen Cui; Stacey, D.A.; 1994, "A modular artificial neural network system for the classification and selection of coatings for a chemical sensor array," Neural Networks,. IEEE World Congress on Computational Intelligence, IEEE International Conference on , pp.3342-3347 vol.5, 27 Jun-2 Jul 1994

[9] Bachtiar, L.R.; Unsworth, C.P.; Newcomb, R.D.; Crampin, E.J.; 2011, "Using artificial neural networks to classify unknown volatile chemicals from the firings of insect olfactory sensory neurons," Engineering in Medicine and Biology Society, EMBC, Annual International Conference of the IEEE , pp.2752-2755, Aug. 30 2011-Sept. 32011

[10] Bachtiar, L.R.; Unsworth, C.P.; Newcomb, R.D.; Crampin, E.J.; 2011, "Predicting odorant chemical class from odorant descriptor values with an assembly of multi-layer perceptrons," Engineering in Medicine and Biology Society, EMBC, Annual International Conference of the IEEE, pp.2756-2759, Aug. 30 2011Sept. 3

[11] Starodub, M.F.; Romanov, V.O.; Kochan, R.V.; Sachenko, A.O.; Kochan, O.V.; 2007, "Implementation of SPR-Biosensors for Express-Diagnostics of Acute Viral Infection and Mycotocsicosis," Medical Measurement and Applications,. MEMEA '07. IEEE International Workshop on , vol., no., pp.1-3, 4-5 May 2007

[12] Slaughter, G.E.; Hobson, R.S.; 2006,"Artificial Neural Network for Temporal Impedance Recognition of Neurotoxins" Neural Networks, IJCNN '06. International Joint Conference on , pp.2001-2008, 0-0 0

[13] Preeti, Dr. H.M. Rai, 2012 "Modeling of Fiber Optic Biosensor to Measure Strain on Bones for Detecting Onset of Osteoporosis", IJSER, vol. 3, no.7. 